\title{
Generating indistinguishable photons from a quantum dot in a noisy environment
}

\author{
Ted S. Santana, ${ }^{1}$ Yong Ma, ${ }^{1, *}$ Ralph N. E. Malein, ${ }^{1}$ Faebian Bastiman, ${ }^{2}$ Edmund Clarke,${ }^{2}$ and Brian D. Gerardot ${ }^{1, \dagger}$ \\ ${ }^{1}$ Institute of Photonics and Quantum Sciences, SUPA, Heriot-Watt University, Edinburgh, United Kingdom \\ ${ }^{2}$ EPSRC National Centre for III-V Technologies, University of Sheffield, Sheffield, United Kingdom \\ (Received 14 December 2016; revised manuscript received 26 April 2017; published 23 May 2017)
}

\begin{abstract}
Single photons from semiconductor quantum dots are promising resources for linear optical quantum computing, or, when coupled to spin states, quantum repeaters. To realize such schemes, the photons must exhibit a high degree of indistinguishability. However, the solid-state environment presents inherent obstacles for this requirement as intrinsic semiconductor fluctuations can destroy the photon indistinguishability. Here, we demonstrate that resonant excitation of a quantum dot with a narrow-band laser generates near transform limited power spectra and indistinguishable photons from a single quantum dot in an environment with many charge-fluctuating traps. The specificity of the resonant excitation suppresses the excited state population in the quantum dot when it is detuned due to spectral fluctuations. The dynamics of this process lead to flickering of the emission over long time scales ( $>5 \mu \mathrm{s}$ ) and reduces the time-averaged count rates. Nevertheless, in spite of significant spectral fluctuations, high visibility two-photon interference can be achieved. This approach is useful for quantum dots with nearby surface states in processed photonic structures and quantum emitters in emerging platforms, such as two-dimensional semiconductors.
\end{abstract}

DOI: 10.1103/PhysRevB.95.201410

\section{INTRODUCTION}

A semiconductor quantum dot (QD) can emulate a twolevel or a multilevel atomic system. Two-level QD transitions can generate single photons with a high degree of indistinguishability [1-13], an ideal resource for implementing future quantum photonic technologies such as boson sampling $[14,15]$ and perhaps ultimately linear optical quantum computing. Multilevel QD systems, including the biexciton $\rightarrow$ exciton $\rightarrow$ ground-state cascade and so-called spin $-\lambda$ systems $[16,17]$ can be used to generate entangled photon pairs [18-21] and spin-photon entanglement [22-24], respectively, which can underpin implementations of quantum repeaters and networks [25]. While a solid-state platform provides benefits for scalability [26], functionality [27,28], and on-chip integration [29-31], inherent fluctuations in the semiconductor matrix act as sources of noise [32-35] that inhomogeneously broaden a QD transition. The inhomogeneous broadening mechanisms can reduce the coherence (with a characteristic dephasing time $T_{2}^{*}$ ) and indistinguishability [with a characteristic two-photon interference visibility $v(0)$ ] of photons extracted from the QD [7,36].

There are two primary inhomogeneous broadening mechanisms for semiconductor QDs at low temperature. Nuclear spin noise, caused by fluctuations in the spin orientation of the QD constituent atoms' nuclei, results in a changing magnetic field that couples to an individual electron or hole spin in the QD. In the context of a single photon source, nuclear spin noise can be mitigated by a few strategies. For the negatively charged trion $\left(X^{1-}\right)$, a modest external magnetic field in the growth direction (Faraday geometry) can screen the nuclear spin fluctuations [12] or a second laser can be

\footnotetext{
*Present address: Chongqing Institute of Green and Intelligent Technology, Chinese Academy of Sciences, Chongqing 400714, China.

†b.d.gerardot@hw.ac.uk
}

used to partially stabilize the Overhauser field [34]. For the neutral exciton $\left(X^{0}\right)$, the effective magnetic field caused by the exchange interaction energy effectively mitigates the nuclear spin noise [12,34]. The second, often more severe, source of inhomogeneous broadening is localized charge fluctuations in the QD environment which dynamically modify the electric field at the position of the QD and alter the dot's transition energy via the Stark effect. Charge noise is present under both incoherent $[3,37,38]$ and coherent $[32,33,39-42]$ excitation. The origins of the charge traps that host the fluctuations can vary depending on the sample; potential sources include nearby surface states in processed photonic structures [43-45], traps created at heterostructure interfaces [41], impurities from intentional dopants [39], and residual background dopant impurities [42,46]. Charge noise is often identified as an origin of increased ensemble dephasing and decreased two-photon interference visibility [3,5,7,31,47].

Although dynamics in the local electric field cannot be universally eliminated in a semiconductor device, the impact of charge noise induced inhomogeneous broadening on photon indistinguishability can be alleviated. One method is to reduce the emitter lifetime $\left(T_{1}\right)$ so that it masks $T_{2}^{*}$, typically achieved with Purcell enhancement by embedding the QD in a highquality factor $(Q)$ cavity [3,47-49]. Unfortunately, increased charge noise often accompanies the Purcell enhancement in processed photonic devices [43-45]. Additionally, recapture of carriers excited by a non- or quasiresonant laser can ruin the purity of the single photon emission from QDs in cavities, especially when driven near saturation [3,10,31,50,51]. An alternative method to suppress the effects of inhomogeneous broadening is to exploit resonantly scattered photons, which offers two significant benefits with respect to $T_{2}^{*}$. First, resonant excitation eliminates populating charge traps via above the band-gap excitation (although ambient charge fluctuations remain). Thus, resonance fluorescence is integral to the generation of single photons with high visibility two-photon interference [8-10,13]. Second, spectral fluctuations occurring at time scales greater than $T_{1}$ which detune the transition 
from the resonant laser by energy $\delta$ effectively increase the fraction of elastically scattered photons at the expense of incoherent scattering [40]. Elastically scattered photons are fundamentally indistinguishable $[4,6]$. Concurrently, the dynamic detuning of the two-level system from the laser resonance leads to fluctuations in extracted photon rates. This flickering has recently been characterized over a wide range of time scales [32-35]. While resonance fluorescence is already established as the leading technique to generate ultrahigh two-photon interference visibility from quantum dots in clean samples with little environmental noise [8-11], here we investigate and demonstrate that highly indistinguishable single photons can be generated from quantum emitters exposed to substantial charge noise. This result can be applied to emitters in less mature, emerging platforms that thus far suffer from more spectral fluctuations than state-of-the-art III-V quantum dot samples. Examples include quantum dots emitting at telecom wavelengths [52] or quantum emitters in two-dimensional materials, for which resonance fluorescence has recently been demonstrated [53].

\section{EXPERIMENTAL SETUP}

Our sample consists of self-assembled InGaAs QDs embedded in a GaAs Schottky diode for deterministic charge state control. The QDs are positioned at an antinode of a fifth-order planar cavity on top of a Au layer which functions as a mirror and Schottky gate [54]. The experiments are performed at $T=4 \mathrm{~K}$ using confocal microscopy. Resonance fluorescence is separated from the reflected laser light using orthogonal linear polarizers in the excitation and collection arms of the confocal microscope [4,32-34]. The resonance fluorescence is detected with a silicon avalanche photodiode (jitter $\sim 500 \mathrm{ps)}$ ) in photon counting mode and the arrival time of each photon is recorded. The resonance fluorescence is further characterized via high-resolution (27 MHz) spectroscopy using a Fabry-Pérot interferometer $(5.5 \mathrm{GHz}$ free spectral range), a Hanbury Brown-Twiss interferometer to measure second-order correlation functions, and an unbalanced MachZender $(\mathrm{MZ})$ interferometer $\left(t_{\text {delay }}=49.7 \mathrm{~ns}\right)$ with polarization control in each arm to measure postselected, two-photon interference.

\section{RESULTS}

\section{A. Photoluminescence and photon statistics}

We first characterize single QDs in the device using nonresonant excitation $(830 \mathrm{~nm})$, confirming bright photoluminescence (PL) and deterministic charging, as shown in Fig. 1(a). Separate measurements reveal that the lifetime of the $X^{1-}$ transition is $625 \mathrm{ps}$, yielding a transform limited linewidth of $1.05 \mu \mathrm{eV}$. Resonance between the driving field and the fluorescence of the QD is then established by setting the laser wavelength constant and sweeping the transition using applied voltage bias $\left(V_{g}\right)$ across resonance. Figure 1(b) shows five such resonance fluorescence spectra from the $X^{1-}$ transition measured in rapid succession. Here, the excitation power is $P_{\text {exc }}=0.029(3) P_{\text {sat }}$, where $P_{\text {sat }}$ is the power at saturation, and the integration time per point is $50 \mathrm{~ms}$. Resonance is randomly achieved within an energy interval of about $10 \mu \mathrm{eV}$
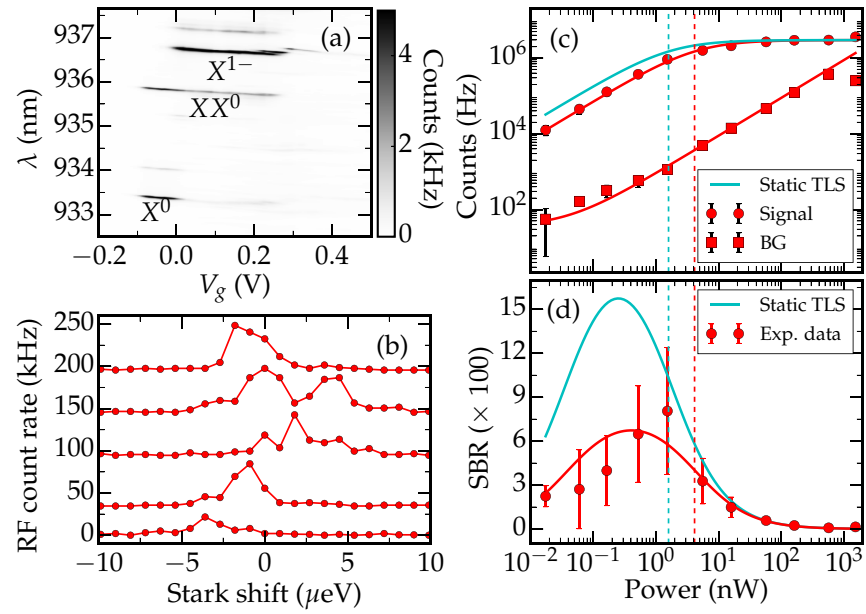

FIG. 1. (a) PL map of an isolated charge tunable QD with the neutral exciton $\left(X^{0}\right)$, the biexciton $\left(X X^{0}\right)$, and the trion $\left(X^{1-}\right)$ states labeled. (b) Five successive resonance fluorescence detuning spectra of the $X^{1-}$ transition with excitation power $P_{\text {exc }}=0.12(1) \mathrm{nW}$ and $50 \mathrm{~ms}$ integration time per data point. Due to charge noise, the transition energy fluctuates. (c) Saturation curve under strong spectral fluctuation (red circle) and the background due to back reflection of the driving field (red square). The expected saturation curve for a static TLS is represented by the cyan line. From the fit (solid red line), the measured saturation power is $P_{\text {sat }}=4.1(3) \mathrm{nW}$ (dashed red line), while the hypothetical static TLS would have $P_{\text {sat }}=1.59 \mathrm{nW}$ (dashed cyan line). (d) Measured signal to background ratio (SBR) under strong spectral fluctuation (red circles) compared with the SBR in the hypothetical case of no spectral fluctuation (cyan line).

due to significant spectral fluctuations. We ascribe this due to a large amount of charge noise in the QD environment that imposes its dynamics on the transition of the QD via the Stark shift. A saturation curve measuring the total number of detected photons $\left(I_{\mathrm{tot}}\right)$ as a function of excitation power is shown in Fig. 1(c). A fit to the curve (solid red line) using $I_{\text {tot }}=\eta \Gamma P_{\text {exc }} / 2\left[P_{\text {exc }}+P_{\text {sat }}\right]$ yields $P_{\text {sat }}=4.1(3) \mathrm{nW}$ and $\eta_{\text {overall }}=0.37(1) \%$, where $\eta$ is the overall efficiency. Accounting for the losses in the beam path and the single photon detector efficiency, the estimated collection of the sample is $\eta_{\text {sample }}=10.8(4) \%$, resulting in more than $90 \times 10^{6}$ photons per second reaching the first lens of the microscope. The cyan curve displays a curve for an ideal, static two-level system (TLS) [55] with $T_{1}=0.625 \mathrm{~ns}$. The saturation power for this static TLS (cyan dashed line) is $1.59 \mathrm{nW}$, approximately three times smaller than the experimental value. Additionally, for excitation powers below saturation, $I_{\text {tot }}$ is reduced relative to the expected value for the static TLS. This impacts the signal to background ratio of the resonance fluorescence [Fig. 1(d)], where the background is the uncancelled reflected laser light. The relatively large error bars are due to charge noise.

The experimental saturation curve is altered because the integration time of $50 \mathrm{~ms}$ in these measurements is long relative to the spectral fluctuation dynamics. This is demonstrated in the time trace measurements acquired by setting $\lambda$ and $V_{g}$ constant and recording the arrival time of the photons in a time tagging mode, shown in Figs. 2(a)-2(c) where the 

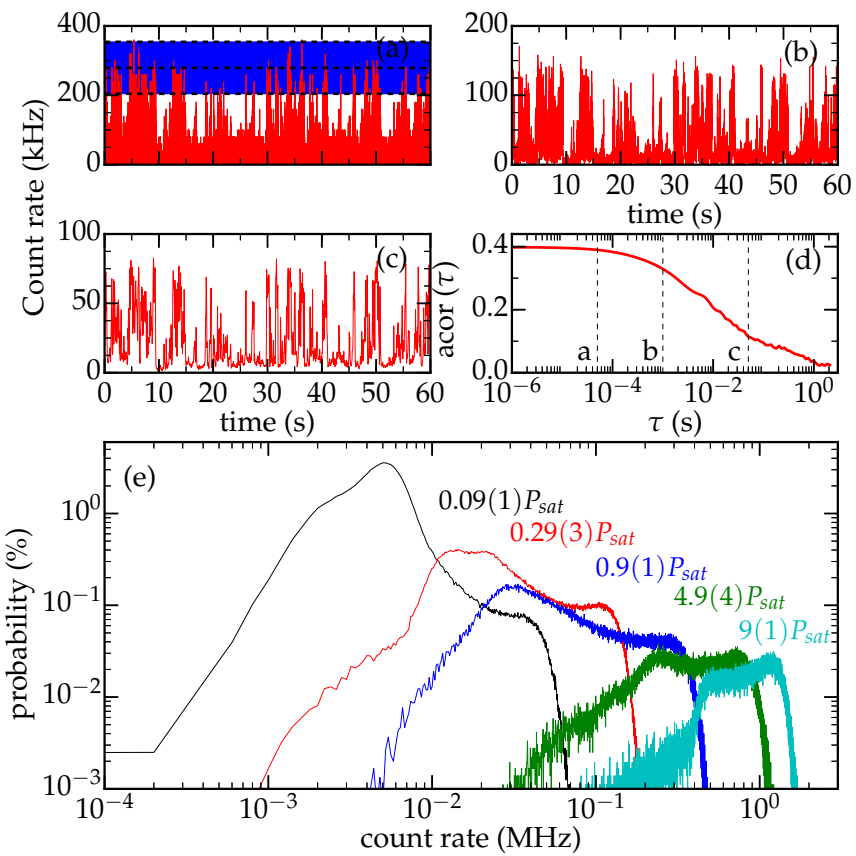

FIG. 2. Time trace of the resonance fluorescence signal for constant $\lambda$ and $V_{g}$ with $P_{\text {exc }}=0.029(3) P_{\text {sat }}$. The shaded area in (a) represents the expected number of photons for an static TLS with a shot noise contribution. The arrival time of the photons were binned using (a) $T_{\text {bin }}=50 \mu \mathrm{s}$, (b) $T_{\text {bin }}=1 \mathrm{~ms}$, and (c) $T_{\text {bin }}=50 \mathrm{~ms}$. (d) Autocorrelation function obtained from the time trace with $T_{\text {bin }}=1 \mu \mathrm{s}$. The dashed lines mark the time bin of the time traces. (e) Empirical probability of counting $I_{\text {tot }}$ photons in a time interval $T_{\text {bin }}=5 \mathrm{~ms}$ for $P_{\mathrm{exc}}=0.09(1) P_{\mathrm{sat}}$ (black), $P_{\mathrm{exc}}=0.29(3) P_{\mathrm{sat}}($ red $)$, $P_{\mathrm{exc}}=0.9(1) P_{\mathrm{sat}}$ (blue), $P_{\mathrm{exc}}=4.9(4) P_{\mathrm{sat}}$ (green), $P_{\mathrm{exc}}=9(1) P_{\mathrm{sat}}$ (cyan).

binning times are $50 \mu \mathrm{s}, 1 \mathrm{~ms}$, and $50 \mathrm{~ms}$, respectively. In Fig. 2(a), the shaded area represents the expected number of photons extracted from an ideal static TLS with the shot noise contribution. The autocorrelation function $\operatorname{acor}(\tau)$ of the time traces presented in Figs. 2(a)-2(c) reveals that the spectral fluctuations have dynamics on the millisecond time scale, as shown in Fig. 2(d). This suggests that the environment of the QD may be considered static in a time interval smaller than $50 \mu \mathrm{s}$, reinforcing previous results [32,33,56,57]. These dynamics confirm that the probable source of spectral fluctuations is a dynamic charge density in the sample. We note that other samples of the identical design grown in different growth chambers do not show these dynamics (e.g., behavior similar to the static TLS is obtained) and therefore conclude that impurities during the growth are the source of the intrinsic charge noise in the sample. The empirical probability of collecting $I_{\text {tot }}$ photons, displayed in Fig. 2(e), is obtained through normalized histogram of the time traces. Because their probability distributions deviate significantly from a Poissonian distribution, the curves in Fig. 2(e) reveal that charge noise still leads to blinking even in the power broadened regime $\left(P>P_{\text {sat }}\right)$. In this case, the power broadening contributes to the decreasing visibility of the off-resonance distribution.
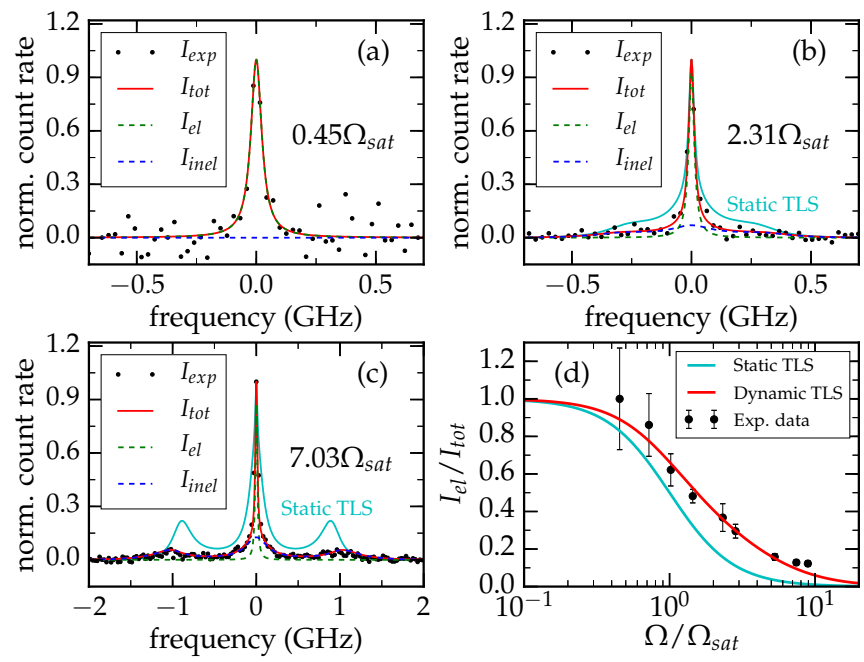

FIG. 3. Power spectrum measurement (black points) fitted with Lorentzian peaks (solid red line), accounting for the elastic scattering (dashed green) and the inelastic scattering (dashed blue) contributions. The solid cyan line [(b), (c)] represents the power spectrum for an ideal static TLS. The Rabi frequencies are (a) $0.45 \Omega_{\text {sat }}$, (b) $2.31 \Omega_{\text {sat }}$, and (c) $7.03 \Omega_{\text {sat }}$. (d) The ratio between the number of elastically scattered photons and the total number of photons (black points) fitted as a TLS under spectral fluctuation (solid red) using $w=2.4(6) \mu \mathrm{eV}$. The solid cyan line represents the ratio $I_{\mathrm{el}} / I_{\mathrm{tot}}$ for an ideal static TLS.

\section{B. Power spectrum}

We now spectrally characterize the resonantly scattered photons from the QD in the noisy environment. Figures 3(a)-3(c) shows high-resolution (27 MHz) spectra from the $X^{1-}$ transition recorded using a scanning FabryPérot interferometer for excitation powers below, near, and above saturation $\left(\Omega_{a}=0.45 \Omega_{\mathrm{sat}}, \Omega_{b}=2.31 \Omega_{\mathrm{sat}}\right.$, and $\Omega_{c}=$ $7.03 \Omega_{\text {sat }}$, respectively). Here, we apply a modest magnetic field $\left(B_{z}^{\text {ext }}=1 \mathrm{~T}\right)$ in the growth direction (Faraday geometry) to screen the effect of nuclear spin fluctuations [12], and excite the highest energy transition. Below saturation, the power spectrum is dominated by elastically scattered photons $\left(I_{\mathrm{el}}\right)$, as expected for a two-level system with negligible pure dephasing. As the Rabi frequency is increased, an inelastic peak $\left(I_{\text {inel }}\right)$ with $\Gamma / 2 \pi \approx 255 \mathrm{MHz}$ gains in intensity. Finally, above saturation, the Mollow triplet consisting of two sidebands (split from the center inelastic peak by the Rabi frequency) as well as the elastic peak are observed. With increasing excitation power, we observe increasing deviation from ideal two-level (static TLS) behavior due to the spectral fluctuations. The sidebands of the Mollow triplet are slightly shifted to higher frequencies and strongly suppressed when compared with the elastic peak. We fit the data by numerically integrating the power spectrum over detuning with a normal distribution as the probability density function (PDF) [Figs. 3(b) and 3(c)], which is a good description for the electric field probability distribution under the assumption of many contributing charge fluctuations observed over a long time period [33]. Figure 3(d) shows the discrepancy between the measured $I_{\mathrm{el}} / I_{\text {tot }}$ (black circles) and the expected curve for a static TLS (solid cyan), which is corrected with 

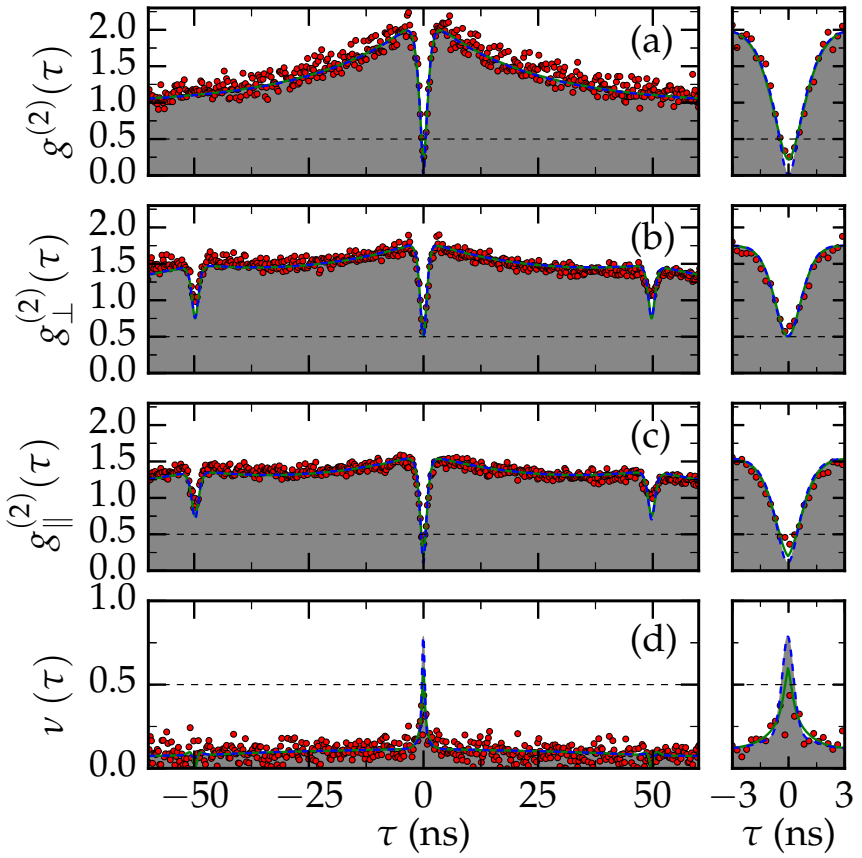

FIG. 4. (a) $g^{(2)}(\tau)$ measurement (red circles) with fitted $g^{(2)}(0)=$ $22 \%$ (solid green) and deconvolved $g^{(2)}(0)=0.1 \%$ (dashed blue). (b), (c) Two-photon interference measurement with the photon polarization at the beam splitter (b) orthogonal or (c) parallel to each other (red circles) with fitted $g_{\perp}^{(2)}(0)=51 \%$ and $g_{\|}^{(2)}(0)=20 \%$ (solid green), and deconvolved $g_{\perp}^{(2)}(0)=50 \%$ and $g_{\|}^{(2)}(0)=11 \%$ (dashed blue). (d) Visibility of the two-photon interference (red circles) with fitted $v(0)=60 \%$ (solid green) and deconvolved $v(0)=79 \%($ dashed blue).

the inclusion of the spectral fluctuations through numerical integration over the detuning with a normal distribution of width $w=2.4(6) \mu \mathrm{eV}$ as the PDF (solid red). We observe that the spectral fluctuations increase the ratio $I_{\mathrm{el}} / I_{\mathrm{tot}}$, in agreement with previous results [40]. Note that the phonon sidebands - which reduce $I_{\mathrm{el}} / I_{\mathrm{tot}}[58]$ - are not observed in the high-resolution spectra and therefore not accounted for in Fig. 3(d). The width of the PDF of the charge noise is below the rough estimate from the five successive detuning spectra in Fig. 1(b) $(\sim 10 \mu \mathrm{eV})$. The origin of this disparity is that photon scattering is limited by the width $L$ of the QD transition (including power broadening effects) while the power spectrum is obtained with a constant $\lambda$ and $V_{g}$. Consequently, when the detuning is greater than $L$, photons are not collected in great quantity and this scenario does not have a significant contribution in the power spectrum. We note that the contribution to the resonance fluorescence signal from the lowest energy $X^{1-}$ transition of the Faraday geometry is negligible due to the large energy separation $(\sim 107 \mu \mathrm{eV})$ compared to spectral fluctuations.

\section{Two-photon interference}

Second-order correlation experiments confirm the single photon nature of the resonance fluorescence signal, as shown in Fig. 4(a). Here, significant bunching around time zero is observed due to optical spin pumping, a consequence of the nonzero external magnetic field [12,59-61]. We use a fitting procedure similar to Malein et al. [12]. Deconvolution of the fit with the response function of the detectors and electronics (dashed blue line) yields $g^{(2)}(0)=0.1 \%$ with $\Omega=0.39 \Omega_{\text {sat }}$. The fitted function gives $g^{(2)}(0)=22 \%$.

In Figs. 4(b) and 4(c) we can observe the two-photon interference measurements when the polarizations of the photons are orthogonal $\left(g_{\perp}^{(2)}\right)$ and parallel $\left(g_{\|}^{(2)}\right)$ to each other, respectively, for $\Omega=0.45 \Omega_{\text {sat }}$. For the distinguishable case, the fit (solid green) and deconvolved curve (dashed blue) give $g_{\perp}^{(2)}(0)$ equal to $51 \%$ and $50 \%$, respectively. When the polarizations are aligned in parallel, the fit gives $g_{\|}^{(2)}(0)=$ $20 \%$, while the minimum value of the deconvolved curve is $11 \%$. The visibility $v(\tau)=\left[g_{\perp}^{(2)}(\tau)-g_{\|}^{(2)}(\tau)\right] / g_{\perp}^{(2)}(\tau)$ is shown in Fig. 4(d) and in the inset (right-hand side panel) with the maximum value of the raw fit and deconvolved curves being $60 \%$ and $79 \%$, respectively. Low-resolution spectra reveal that $\sim 10 \%$ of the power spectrum is due to photons scattered into phonon sidebands. These incoherently scattered photons, not filtered in the two-photon interference experiment, are expected to reduce the maximum possible visibility to $\sim 80 \%$. Therefore, we conclude the strong spectral fluctuations due to charge noise do not degrade the indistinguishability of successively emitted photons within the $50 \mathrm{~ns}$ time delay used in the unbalanced $\mathrm{MZ}$ interferometer.

\section{SUMMARY}

We have presented the photon emission statistics from a quantum dot undergoing strong spectral fluctuations due to a large number of charge traps in its environment. In our device, the fluctuating charges-with dynamics in the millisecond time scale-cause a Stark shift up to $\sim 10 \mu \mathrm{eV}$ even with resonant excitation. This leads to flickering in intensity which decreases the time-averaged count rates for excitation powers below saturation and, consequently, the signal to background ratio. Compared to an ideal, static two-level system, the resonance fluorescence power spectra exhibit shifted and inhibited Rabi sidebands. Importantly, the central peak experiences negligible inhomogeneous broadening due to the spectral fluctuations. Rather, the ratio of elastic to inelastic scattered light is affected by the charge noise. Finally, two-photon interference experiments show that photons generated within a short time delay (50 ns) are highly coherent and indistinguishable. We conclude that, in spite of the flickering intensity due to spectral fluctuations, the resonance fluorescence technique enables the generation of highly coherent and indistinguishable photons from two-level quantum emitters in a noisy environment.

\section{ACKNOWLEDGMENTS}

This work was supported by the EPSRC (Grants No. EP/I023186/1, No. EP/K015338/1, No. EP/M013472/1, and No. EP/G03673X/1) and by ERC Starting Grant No. 307392. B.D.G. thanks the Royal Society for a University Research Fellowship. 
[1] R. B. Patel, A. J. Bennett, K. Cooper, P. Atkinson, C. A. Nicoll, D. A. Ritchie, and A. J. Shields, Phys. Rev. Lett. 100, 207405 (2008).

[2] S. Ates, S. M. Ulrich, S. Reitzenstein, A. Löffler, A. Forchel, and P. Michler, Phys. Rev. Lett. 103, 167402 (2009).

[3] O. Gazzano, S. M. de Vasconcellos, C. Arnold, A. Nowak, E. Galopin, I. Sagnes, L. Lanco, A. Lemaître, and P. Senellart, Nat. Commun. 4, 1425 (2013).

[4] C. Matthiesen, M. Geller, C. H. Schulte, C. Le Gall, J. Hansom, Z. Li, M. Hugues, E. Clarke, and M. Atatüre, Nat. Commun. 4, 1600 (2013).

[5] L. Monniello, A. Reigue, R. Hostein, A. Lemaitre, A. Martinez, R. Grousson, and V. Voliotis, Phys. Rev. B 90, 041303 (2014).

[6] R. Proux, M. Maragkou, E. Baudin, C. Voisin, P. Roussignol, and C. Diederichs, Phys. Rev. Lett. 114, 067401 (2015).

[7] A. Thoma, P. Schnauber, M. Gschrey, M. Seifried, J. Wolters, J.-H. Schulze, A. Strittmatter, S. Rodt, A. Carmele, A. Knorr et al., Phys. Rev. Lett. 116, 033601 (2016).

[8] N. Somaschi, V. Giesz, L. De Santis, J. C. Loredo, M. P. Almeida, G. Hornecker, S. L. Portalupi, T. Grange, C. Anton, J. Demory et al., Nat. Photonics 10, 340 (2016).

[9] X. Ding, Y. He, Z.-C. Duan, N. Gregersen, M.-C. Chen, S. Unsleber, S. Maier, C. Schneider, M. Kamp, S. Höfling, C.-Y. Lu, and J.-W. Pan, Phys. Rev. Lett. 116, 020401 (2016).

[10] T. Huber, A. Predojević, D. Föger, G. Solomon, and G. Weihs, New J. Phys. 17, 123025 (2015).

[11] A. C. Dada, T. S. Santana, R. N. E. Malein, A. Koutroumanis, Y. Ma, J. M. Zajac, J. Y. Lim, J. D. Song, and B. D. Gerardot, Optica 3, 493 (2016).

[12] R. N. E. Malein, T. S. Santana, J. M. Zajac, A. C. Dada, E. M. Gauger, P. M. Petroff, J. Y. Lim, J. D. Song, and B. D. Gerardot, Phys. Rev. Lett. 116, 257401 (2016).

[13] S. Unsleber, Y.-M. He, S. Gerhardt, S. Maier, C.-Y. Lu, J.-W. Pan, N. Gregersen, M. Kamp, C. Schneider, and S. Höfling, Opt. Express 24, 8539 (2016).

[14] J. C. Loredo, M. A. Broome, P. Hilaire, O. Gazzano, I. Sagnes, A. Lemaitre, M. P. Almeida, P. Senellart, and A. G. White, Phys. Rev. Lett. 118, 130503 (2017).

[15] H. Wang, Y. He, Y.-H. Li, Z.-E. Su, B. Li, H.-L. Huang, X. Ding, M.-C. Chen, C. Liu, J. Qin et al., Nat. Photon. (2017), doi:10.1038/nphoton.2017.63.

[16] X. Xu, B. Sun, E. D. Kim, K. Smirl, P. R. Berman, D. G. Steel, A. S. Bracker, D. Gammon, and L. J. Sham, Phys. Rev. Lett. 101, 227401 (2008)

[17] D. Brunner, B. D. Gerardot, P. A. Dalgarno, G. Wüst, K. Karrai, N. G. Stoltz, P. M. Petroff, and R. J. Warburton, Science 325, 70 (2009).

[18] N. Akopian, N. H. Lindner, E. Poem, Y. Berlatzky, J. Avron, D. Gershoni, B. D. Gerardot, and P. M. Petroff, Phys. Rev. Lett. 96, 130501 (2006).

[19] C. L. Salter, R. M. Stevenson, I. Farrer, C. A. Nicoll, D. A. Ritchie, and A. J. Shields, Nature (London) 465, 594 (2010).

[20] R. Trotta, J. Martín-Sánchez, J. S. Wildmann, G. Piredda, M. Reindl, C. Schimpf, E. Zallo, S. Stroj, J. Edlinger, and A. Rastelli, Nat. Commun. 7, 10375 (2016).

[21] Y. Chen, J. Zhang, M. Zopf, K. Jung, Y. Zhang, R. Keil, F. Ding, and O. G. Schmidt, Nat. Commun. 7, 10387 (2016).
[22] W. B. Gao, P. Fallahi, E. Togan, A. Delteil, Y. S. Chin, J. MiguelSanchez, and A. Imamoğlu, Nat. Commun. 4, 2744 (2013).

[23] K. De Greve, L. Yu, P. L. McMahon, J. S. Pelc, C. M. Natarajan, N. Y. Kim, E. Abe, S. Maier, C. Schneider, M. Kamp et al., Nature (London) 491, 421 (2012).

[24] J. R. Schaibley, A. P. Burgers, G. A. McCracken, L.-M. Duan, P. R. Berman, D. G. Steel, A. S. Bracker, D. Gammon, and L. J. Sham, Phys. Rev. Lett. 110, 167401 (2013).

[25] T. Northup and R. Blatt, Nat. Photonics 8, 356 (2014).

[26] K. D. Jons, P. Atkinson, M. Muller, M. Heldmaier, S. M. Ulrich, O. G. Schmidt, and P. Michler, Nano Lett. 13, 126 (2012).

[27] R. J. Warburton, C. Schäflein, D. Haft, F. Bickel, A. Lorke, K. Karrai, J. M. Garcia, W. Schoenfeld, and P. M. Petroff, Nature (London) 405, 926 (2000).

[28] R. Trotta, P. Atkinson, J. D. Plumhof, E. Zallo, R. O. Rezaev, S. Kumar, S. Baunack, J. R. Schröter, A. Rastelli, and O. G. Schmidt, Adv. Mater. 24, 2668 (2012).

[29] I. J. Luxmoore, N. A. Wasley, A. J. Ramsay, A. C. T. Thijssen, R. Oulton, M. Hugues, S. Kasture, V. G. Achanta, A. M. Fox, and M. S. Skolnick, Phys. Rev. Lett. 110, 037402 (2013).

[30] S. Kalliakos, Y. Brody, A. Schwagmann, A. J. Bennett, M. B. Ward, D. J. P. Ellis, J. Skiba-Szymanska, I. Farrer, J. P. Griffiths, G. A. C. Jones et al., Appl. Phys. Lett. 104, 221109 (2014).

[31] K. H. Madsen, S. Ates, J. Liu, A. Javadi, S. M. Albrecht, I. Yeo, S. Stobbe, and P. Lodahl, Phys. Rev. B 90, 155303 (2014).

[32] A. V. Kuhlmann, J. Houel, A. Ludwig, L. Greuter, D. Reuter, A. D. Wieck, M. Poggio, and R. J. Warburton, Nat. Phys. 9, 570 (2013).

[33] C. Matthiesen, M. J. Stanley, M. Hugues, E. Clarke, and M. Atatüre, Sci. Rep. 4, 4911 (2014).

[34] A. V. Kuhlmann, J. H. Prechtel, J. Houel, A. Ludwig, D. Reuter, A. D. Wieck, and R. J. Warburton, Nat. Commun. 6, 8204 (2015).

[35] M. J. Stanley, C. Matthiesen, J. Hansom, C. Le Gall, C. H. H. Schulte, E. Clarke, and M. Atatüre, Phys. Rev. B 90, 195305 (2014).

[36] T. Legero, T. Wilk, A. Kuhn, and G. Rempe, Appl. Phys. B 77, 797 (2003)

[37] H. D. Robinson and B. B. Goldberg, Phys. Rev. B 61, R5086 (2000).

[38] A. Berthelot, I. Favero, G. Cassabois, C. Voisin, C. Delalande, P. Roussignol, R. Ferreira, and J.-M. Gérard, Nat. Phys. 2, 759 (2006).

[39] B. D. Gerardot, R. J. Barbour, D. Brunner, P. A. Dalgarno, A Badolato, N. Stoltz, P. M. Petroff, J. Houel, and R. J. Warburton, Appl. Phys. Lett. 99, 243112 (2011).

[40] K. Konthasinghe, J. Walker, M. Peiris, C. K. Shih, Y. Yu, M. F. Li, J. F. He, L. J. Wang, H. Q. Ni, Z. C. Niu et al., Phys. Rev. B 85, 235315 (2012)

[41] J. Houel, A. V. Kuhlmann, L. Greuter, F. Xue, M. Poggio, B. D. Gerardot, P. A. Dalgarno, A. Badolato, P. M. Petroff, A. Ludwig et al., Phys. Rev. Lett. 108, 107401 (2012).

[42] M. Hauck, F. Seilmeier, S. E. Beavan, A. Badolato, P. M. Petroff, and A. Högele, Phys. Rev. B 90, 235306 (2014).

[43] C. F. Wang, A. Badolato, I. Wilson-Rae, P. M. Petroff, E. Hu, J. Urayama, and A. Imamoğlu, Appl. Phys. Lett. 85, 3423 (2004).

[44] A. Majumdar, E. D. Kim, and J. Vučković, Phys. Rev. B 84, 195304 (2011).

[45] M. Davanço, C. S. Hellberg, S. Ates, A. Badolato, and K. Srinivasan, Phys. Rev. B 89, 161303 (2014). 
[46] H. S. Nguyen, G. Sallen, M. Abbarchi, R. Ferreira, C. Voisin, P. Roussignol, G. Cassabois, and C. Diederichs, Phys. Rev. B 87, 115305 (2013).

[47] C. Santori, D. Fattal, J. Vučković, G. S. Solomon, and Y. Yamamoto, Nature (London) 419, 594 (2002).

[48] S. Laurent, S. Varoutsis, L. Le Gratiet, A. Lemaître, I. Sagnes, F. Raineri, A. Levenson, I. Robert-Philip, and I. Abram, Appl. Phys. Lett. 87, 163107 (2005).

[49] T. Grange, G. Hornecker, D. Hunger, J.-P. Poizat, J.-M. Gérard, P. Senellart, and A. Auffèves, Phys. Rev. Lett. 114, 193601 (2015).

[50] S. Strauf, N. G. Stoltz, M. T. Rakher, L. A. Coldren, P. M. Petroff, and D. Bouwmeester, Nat. Photonics 1, 704 (2007).

[51] E. B. Flagg, S. V. Polyakov, T. Thomay, and G. S. Solomon, Phys. Rev. Lett. 109, 163601 (2012).

[52] R. Al-Khuzheyri, A. C. Dada, J. Huwer, T. S. Santana, J. Skiba-Szymanska, M. Felle, M. B. Ward, R. M. Stevenson, I. Farrer, M. G. Tanner, R. H. Hadfield, D. A. Ritchie, A. J. Shields, and B. D. Gerardot, Appl. Phys. Lett. 109, 163104 (2016).
[53] S. Kumar, M. Brotóns-Gisbert, R. Al-Khuzheyri, A. Branny, G. Ballesteros-Garcia, J. F. Sánchez-Royo, and B. D. Gerardot, Optica 3, 882 (2016).

[54] Y. Ma, P. E. Kremer, and B. D. Gerardot, J. Appl. Phys. 115, 023106 (2014).

[55] B. R. Mollow, Phys. Rev. 188, 1969 (1969).

[56] J. H. Prechtel, A. V. Kuhlmann, J. Houel, L. Greuter, A. Ludwig, D. Reuter, A. D. Wieck, and R. J. Warburton, Phys. Rev. X 3, 041006 (2013).

[57] J. Hansom, C. H. H. Schulte, C. Matthiesen, M. J. Stanley, and M. Atatüre, Appl. Phys. Lett. 105, 172107 (2014).

[58] J. Iles-Smith, D. P. McCutcheon, J. Mørk, and A. Nazir, arXiv:1606.06305.

[59] X. Xu, Y. Wu, B. Sun, Q. Huang, J. Cheng, D. G. Steel, A. S. Bracker, D. Gammon, C. Emary, and L. J. Sham, Phys. Rev. Lett. 99, 097401 (2007).

[60] J. Hansom, C. H. H. Schulte, C. Le Gall, C. Matthiesen, E. Clarke, M. Hugues, J. M. Taylor, and M. Atatüre, Nat. Phys. 10, 725 (2014).

[61] J. Dreiser, M. Atatüre, C. Galland, T. Müller, A. Badolato, and A. Imamoglu, Phys. Rev. B 77, 075317 (2008). 